\title{
EFEITO DO TRATAMENTO TÉRMICO NA MICROESTRUTURA, TURBOSTRATICIDADE E SUPERFÍCIE DE CARBONO VÍTREO RETICULADO ANALISADO POR XPS, ESPALHAMENTO RAMAN E VOLTAMETRIA CÍCLICA
}

\author{
Emerson Sarmento Gonçalves e Mirabel Cerqueira Rezende \\ Divisão de Materiais, Instituto de Aeronáutica e Espaço, Centro Técnico Aeroespacial, Praça Marechal Eduardo Gomes, 50, \\ 12228-904, São José dos Campos - SP, Brasil \\ Maurício Ribeiro Baldan e Neidenêi Gomes Ferreira* \\ Centro de Tecnologias Especiais, Instituto Nacional de Pesquisas Espaciais, Av. dos Astronautas, 1758, 12245-970 São José dos \\ Campos - SP, Brasil
}

Recebido em 31/3/08; aceito em 11/8/08; publicado na web em 18/12/08

\begin{abstract}
THERMAL TREATMENT EFFECT ON THE MICROSTRUCTURE, TURBOSTRATICITY, AND SURFACE OF RETICULATED VITREOUS CARBON ANALYZED BY XPS, RAMAN SCATTERING, AND CYCLIC VOLTAMMETRY. The structural and surface properties of reticulated vitreous carbon (RVC) were discussed as a function of its heat treatment temperature (HTT), for samples produced in the range from 700 to $2000{ }^{\circ} \mathrm{C}$, using the furfuryl precursor resin. The samples were analyzed by x-ray photoelectron spectroscopy, first and second order Raman scattering as well as electrochemical response. Exploring the material turbostraticity concept, the interdependence between the RVC chemical surface variation and its defects were demonstrated. The influence of heteroatom presence was discussed in the material ordering for HTT lower than $1300^{\circ} \mathrm{C}$ while the graphitization process evolution was also pointed out for HTT higher than $1500^{\circ} \mathrm{C}$.
\end{abstract}

Keywords: reticulated vitreous carbon; Raman scattering; x-ray photoelectron spectroscopy.

\section{INTRODUÇÃO}

O carbono vítreo reticulado (CVR) é uma estrutura de poros abertos do tipo colméia, que pertence à classe dos carbonos turbostráticos, apresentando estrutura carbonosa lamelar, semelhante à grafite, com hibridização $\mathrm{sp}^{2}$, formando fitas que se arranjam ao acaso no espaço. O CVR apresenta propriedades semicondutoras, reduzida densidade, expansão térmica inexpressiva, alta resistência à corrosão, coeficiente de expansão térmica igual ao da grafite artificial e a condutividade térmica é um pouco menor. A resistência ao choque térmico é somente um décimo da resistência da grafite devido à menor condutividade térmica, em adição ao seu alto grau de dureza e alto valor de módulo. O CVR também apresenta a menor resistividade elétrica específica entre os materiais carbonosos, em torno de $40 \times 10^{-6} \Omega \mathrm{m} .{ }^{1}$ Devido às matrizes porosas usadas como substratos de ancoragem para o CVR, este apresenta de 96 a $98 \%$ do volume consistindo em poros abertos.

Diversas são as aplicações possíveis para este material, especialmente em saúde pública, como, por exemplo, na confecção de próteses ósseas, biossensores para determinação de enzimas e outros compostos presentes no metabolismo, bem como na avaliação da toxicidade de águas e soluções orgânicas. ${ }^{2-4}$ Seu uso na forma monolítica também se destaca na produção de válvulas cardíacas. ${ }^{1}$ Essas aplicações se devem à sua adequada biocompatibilidade. ${ }^{2} \mathrm{O}$ carbono vítreo é um material ímpar para trabalhar em ambientes agressivos, como meios muito oxidantes ou temperaturas elevadas (superiores a $400^{\circ} \mathrm{C}$ em ambiente inerte). Neste contexto, o CVR é um material útil na preparação de eletrodos, particularmente onde são necessárias: altas densidades de corrente $\left(10^{2}\right.$ a $\left.10^{3} \mathrm{~mA} \mathrm{~cm}^{-2}\right)$, baixas resistências ao fluxo elétrico (inferiores a $1 \Omega \mathrm{cm}$ ) e de fluidos e máxima disponibilidade de volume para percolação de fluidos, acima de

*e-mail: neidenei@las.inpe.br
$90 \%$, como ocorre com esse material. ${ }^{5}$ Este trabalho está motivado pelo necessário entendimento das propriedades físico-químicas dos materiais carbonosos processados a diferentes temperaturas de tratamento térmico e como estas propriedades podem influenciar em suas diferentes aplicações.

Embora o carbono vítreo seja um material já há muito utilizado em grande escala e comercialmente disponível no mercado, um estudo sistemático da dependência de suas propriedades com os parâmetros de processamento e temperatura, bem como do material precursor do mesmo ainda se faz necessário. Além disso, a interdependência entre todos esses parâmetros não está completamente elucidada na literatura, principalmente relacionando-a aos efeitos de superfície sempre presentes quando materiais carbonosos são utilizados como eletrodos. Como a técnica Raman é muito sensível a pequenas variações na desordem estrutural, revela-se muito apropriada na análise de várias formas de carbono amorfo. Entretanto, a interpretação dos espectros Raman de materiais carbonosos tem gerado debates consideráveis na literatura, principalmente, devido às complicadas microestruturas desses materiais e à abundância de informações que podem ser obtidas de seus espectros. Diversos autores relacionam a presença e a intensidade relativa da banda de defeitos às propriedades eletroquímicas de eletrodos carbonosos. ${ }^{6,7}$ É importante salientar que a formação de defeitos estruturais em grafite pirolítico altamente ordenado (HOPG), induzida através de ativação a laser, mostrou que as alterações na capacitância e na constante cinética de transferência de elétrons desse material estavam relacionadas às transformações ocorridas na microestrutura carbonosa.

Entre outros estudos importantes, ${ }^{8,9}$ McDermott et al. ${ }^{10}$ caracterizaram a superfície do plano basal de HOPG e examinaram suas propriedades eletroquímicas. A perda de simetria e o surgimento de novos fônons de vibração da rede do HOPG, devido a defeitos nos planos de borda, pelas mais diversas causas, proporcionaram a formação de sítios na superfície. Esses autores concluíram que a 
capacitância, a constante heterogênea de transferência de elétrons $k^{0}$ e a adsorção freqüentemente variam mais significativamente em função da proporção de planos de borda e basais na superfície exposta. Nesse caso, o carbono vítreo apresenta cinética mais rápida que os planos basais do HOPG, devido sua maior densidade de planos de borda. Além disso, qualquer medida das propriedades inerentes ao plano basal é provavelmente mascarada pela influência dos defeitos do plano de borda, e este problema aumenta com o aumento da anisotropia do material.

Este trabalho discute e relaciona o efeito da temperatura de processamento do CVR com suas propriedades físico-químicas de superfície, analisadas por espectroscopia de fotoelétrons por raios-X (X-Ray Photoelectron Spectroscopy, XPS), espectroscopia de espalhamento Raman e resposta eletroquímica por voltametria cíclica (VC) em par redox ferri/ferrocianeto. Trabalhos anteriores já foram publicados com contribuições parciais desse amplo estudo também destacando aspectos importantes e que serão referendados, quando necessário, durante a discussão do artigo. ${ }^{11-15}$ No entanto, a principal contribuição que este texto se propõe é relacionar os dados de XPS com espectroscopia Raman de primeira e segunda ordem, de forma sistemática, em um largo intervalo de temperatura de tratamento térmico (Heat Treatment Temperature, HTT) para a obtenção do CVR entre 700 e $2000{ }^{\circ} \mathrm{C}$, também retomando conceitos fundamentais de turbostraticidade, tão essenciais na explicação dos resultados. Adicionalmente, os resultados da variação dos grupos funcionais de superfície do CVR, em função do tratamento térmico, estão corroborados com a resposta eletroquímica $\mathrm{VC}$, onde claramente está confirmada pela presença de heteroátomos e defeitos nestas estruturas carbonosas que podem ser determinantes em seu desempenho nas diversas aplicações propostas na literatura atual.

\section{PARTE EXPERIMENTAL}

Para o processamento do CVR utilizou-se a espuma CA-120 da Sanko Espumas Ltda., com 70 ppi. Trata-se de uma espuma de poliuretana produzida a partir de poliol poliéter. A resina furfurílica, fornecida pela empresa HP Biopróteses Ltda. de São Paulo/SP, foi misturada com catalisador para acelerar o processo de cura. Foi usado o ácido p-toluenossulfônico (APTS), na proporção de 5\% em massa da quantidade total de resina. A cura completa da resina ocorreu por tratamento térmico em estufa, de acordo com o seguinte ciclo térmico: $2 \mathrm{~h}$ a $90^{\circ} \mathrm{C}$, seguida de mais $2 \mathrm{~h}$ a $130^{\circ} \mathrm{C}$.

O tratamento térmico de carbonização para a obtenção do carbono vítreo reticulado se dá elevando-se a temperatura das amostras a temperaturas acima de $700^{\circ} \mathrm{C}$. Este procedimento foi realizado tendo como temperaturas máximas 700, 1000, 1300, 1500, 1800 e $2000^{\circ} \mathrm{C}$, para cada conjunto de amostras de espumas impregnadas com resina, em forno tubular, programado para aquecer a $60{ }^{\circ} \mathrm{C} \mathrm{h}^{-1}$, a partir da temperatura ambiente, em atmosfera de nitrogênio com fluxo de $1 \mathrm{~L}$ $\mathrm{h}^{-1}$. Ao atingir a temperatura máxima desejada fez-se um patamar por $30 \mathrm{~min}$. Em seguida, o forno é resfriado naturalmente. Este procedimento foi o mesmo para todos os tratamentos térmicos até $1000^{\circ} \mathrm{C}$. Para amostras tratadas termicamente em temperaturas superiores a $1000{ }^{\circ} \mathrm{C}$, após o resfriamento estas são transferidas para um segundo forno para um pós-tratamento térmico, que acontece com taxa de aquecimento de $600{ }^{\circ} \mathrm{C} \mathrm{h}^{-1}$, também com fluxo de nitrogênio.

$\mathrm{O}$ estudo de grupos funcionais de superfície foi realizado a partir das análises de XPS, empregando-se o espectromicroscópio de superfícies (Kratos XSAM HS), em ambiente de ultra-alto vácuo (pressão na faixa de $10^{-8}$ Torr). Para isto, foi empregada como fonte excitadora a radiação $\mathrm{K}$ do alumínio, com energia de $1486,6 \mathrm{eV}$ e potência de $156 \mathrm{~W}$, dada pela voltagem de $13 \mathrm{kV}$ e emissão de $12 \mathrm{~mA}$. Como referência de energia de ligação foi usado o valor $284,8 \mathrm{eV}$ para a linha fotoelétrica $\mathrm{C} 1 \mathrm{~s}$ associada a C-C e/ou C-H. Os espectros foram ajustados utilizando uma linha de base "Shirley" e o procedimento padrão para análise de XPS na deconvolução dos picos, mantendo sempre com parâmetro de ajuste o $\chi^{2}$ próximo da unidade. Esse ajuste pode ser feito tanto pelo programa fornecido pelo fabricante do equipamento ou utilizando um programa gráfico disponível que atenda os requisitos para XPS. Os percentuais relativos às energias das ligações apresentadas na Tabela 1 foram obtidos diretamente a partir das áreas das curvas deconvoluídas conforme mostrados nos gráficos dos picos apresentados. Um sistema de micro-Raman Renishaw 2000, à temperatura ambiente empregando um laser de argônio a 514,5 nm, de $10 \mathrm{~mW}$ de potência, foi usado para obter os espectros de primeira e segunda ordem. Os espectros foram obtidos na configuração backscattering utilizando uma objetiva com spot size de diâmetro $10 \mu \mathrm{m}$. Para cada amostra de CVR foram realizadas 5 varreduras em 5 posições diferentes, utilizando sempre a mesma potência incidente do laser. Lembrando que como as amostras são porosas, foram escolhidas as posições referentes a cada haste subseqüente, procurando manter o mesmo plano focal. Todos os espectros foram ajustados e os desvios foram obtidos pelo desvio quadrático da média das medidas realizadas.

As análises voltamétricas foram realizadas em triplicata fazendose o contato elétrico através de uma haste de cobre. Os eletrodos não tiveram pré-tratamento de superfície antes das medidas de voltametria cíclica. Estas foram realizadas utilizando-se um potenciostato Autolab PGSTAT 302, usando $1 \mathrm{mM}$ de ferrocianeto/0,1 M KCl em taxas de varredura de 5 a $500 \mathrm{mV} \mathrm{s}^{-1}$. Os experimentos foram realizados à temperatura ambiente em uma célula simples de compartimento único e três eletrodos (WE - eletrodo de trabalho de CVR, CE - contraeletrodo de tela de platina, $\mathrm{RE}$ - eletrodo de referência de $\mathrm{Ag} / \mathrm{AgCl}$ ). A solução não foi agitada durante os experimentos voltamétricos.

\section{RESULTADOS E DISCUSSÃO}

\section{Análise de superfície por espectroscopia fotoeletrônica de raios X (XPS)}

Essa técnica foi importante na identificação e quantificação de heteroátomos e tipos de ligações químicas existentes na superfície do material. Além disso, viabilizou o entendimento sobre o processo de migração atômica que acontece à medida que se processa o tratamento térmico. Os espectros das amostras CVR tratadas entre 700 e $2000{ }^{\circ} \mathrm{C}$ mostraram que as quantidades de nitrogênio e oxigênio presentes são suficientes para registrar diferenças significativas no aspecto estrutural do material. Assim, esses grupos funcionais necessitam serem determinados e quantificados, pois estão associados à presença de defeitos, além de variações na sua acidez, o que lhes conferirá caráter catódico ou anódico, a depender da distribuição dos grupos. As bandas mais intensas, em torno de $285,0 \mathrm{eV}$, são típicas do átomo de carbono; as que se localizam em torno de 400,0 eV se referem ao átomo de nitrogênio; e as demais, em torno de $530,0 \mathrm{eV}$, são bandas relacionadas aos grupos oxigenados. ${ }^{16}$ As duas últimas bandas destacam-se, especialmente, quando a amostra é tratada em temperatura abaixo de $1000{ }^{\circ} \mathrm{C}$. Essas bandas foram todas deconvoluídas para uma melhor análise. Para o CVR 700, a Figura 1 mostra a região dos grupos oxigenados (Figura 1a) e para o nitrogênio (Figura 1b). Na Figura 2 está somente representada a região deconvoluída para os grupos oxigenados da amostra CVR 1000, uma vez que nesta temperatura já não se pode mais observar as bandas em torno de 400,0 $\mathrm{eV}$, referentes ao nitrogênio. As bandas típicas do átomo de carbono estão presentes em todos os espectros e suas particularidades para cada temperatura serão discutidas no decorrer das análises.

$\mathrm{O}$ pico $\mathrm{C} 1 \mathrm{~s}$ tem duas contribuições que podem ser associadas a 

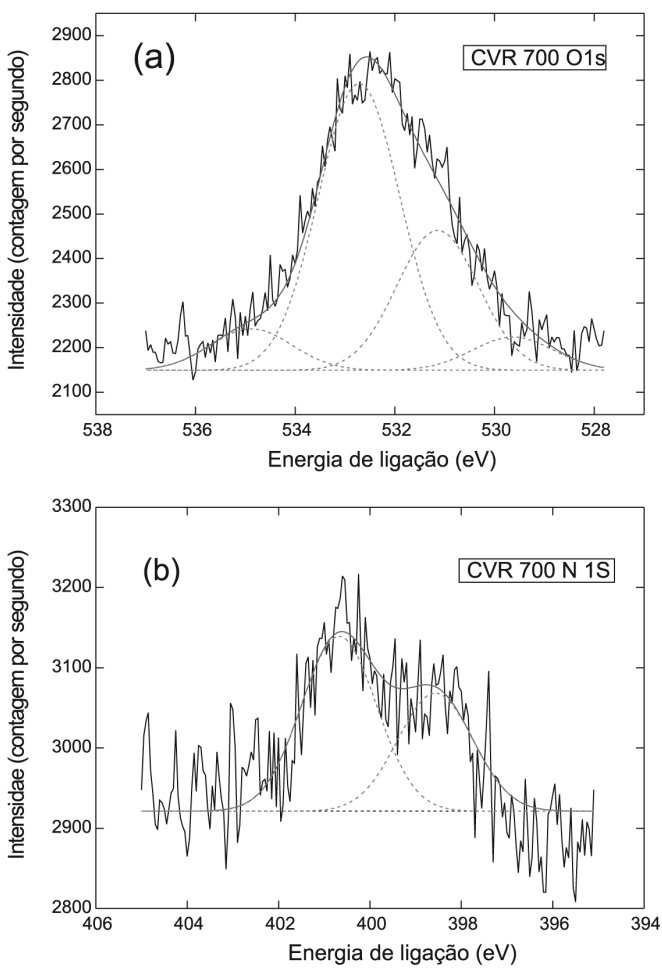

Figura 1. Espectros de XPS do CVR 700. (a) banda de grupos oxigenados; (b) banda de grupos nitrogenados

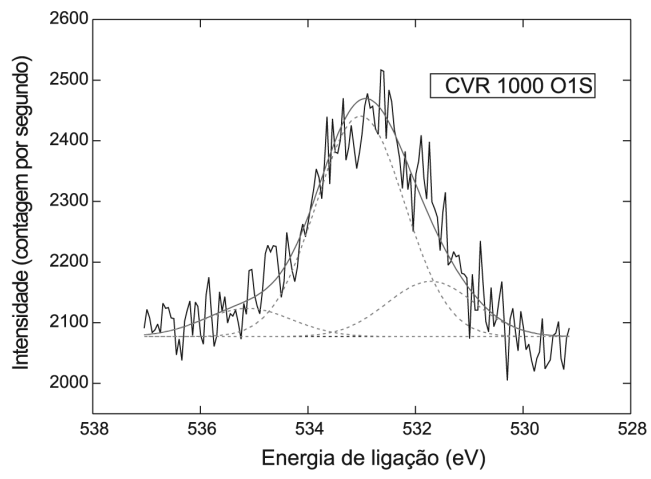

Figura 2. Espectro de XPS do CVR 1000 na região da banda de grupos oxigenados

quatro ligações químicas possíveis: uma corresponde a $\mathrm{C}-\mathrm{C}$ ou $\mathrm{C}-\mathrm{H}$, a 284,8 eV; a outra aproximadamente a 286 eV, a C-O e/ou C-N. Além disso, uma terceira contribuição foi observada a $288,2 \mathrm{eV}$, referente a $\mathrm{O}-\mathrm{C}-\mathrm{O}, \mathrm{C}=\mathrm{O}$ e/ou $\mathrm{C}-\mathrm{N}$. Especificamente, para a amostra tratada a $700{ }^{\circ} \mathrm{C}$, a contribuição a $283,4 \mathrm{eV}$ pode corresponder a um carbeto. $\mathrm{O}$ pico $\mathrm{O} 1 \mathrm{~s}$ pode ser deconvoluído em três ou quatro componentes (Figura 1a). $\mathrm{O}$ componente que corresponde a $\mathrm{C}=\mathrm{O}$ aparece na faixa de 531 a $532 \mathrm{eV}$, e o associado a C-O a 532,0-533,3 eV. Os componentes com menores energias de ligação correspondem a óxidos metálicos e os de maior energia de ligação, a -OH.

Para a amostra de CVR tratada a $700{ }^{\circ} \mathrm{C}$, o pico N 1s (Figura 1b) apresenta-se com dois componentes. Vários autores associaram a componente de energia mais baixa à ligação $\mathrm{sp}^{3} \mathrm{C}-\mathrm{N}$, enquanto a componente com energia acima de $400 \mathrm{eV}$, à ligação $\mathrm{sp}^{2} \mathrm{C}-\mathrm{N} \cdot \cdot^{17,18}$ Entretanto, outros autores contestam esta atribuição, alegando que a componente com menor energia de ligação pode estar associada a um átomo de nitrogênio do piridínico ligado a uma vacância. ${ }^{19}$ Os resultados mostrados na Tabela 1 revelam uma migração de
Tabela 1. Valores das energias de ligação (em eV) dos componentes dos principais picos fotoelétricos. As percentagens em parênteses referem-se às quantidades relativas de cada componente de um determinado pico

\begin{tabular}{lccc}
\hline $\begin{array}{l}\text { Temperatura } \\
\text { de Tratamento } \\
\text { Térmico }\left({ }^{\circ} \mathrm{C}\right)\end{array}$ & C 1s & O 1s & N 1s \\
\hline & $283,4(3 \%)$ & $529,8(6 \%)$ & $398,4(42 \%)$ \\
700 & $284,8(74 \%)$ & $531,4(28 \%)$ & $400,6(58 \%)$ \\
& $286,3(18 \%)$ & $532,8(59 \%)$ & \\
& $288,2(5 \%)$ & $534,5(7 \%)$ & \\
1000 & $284,8(81 \%)$ & $531,7(19 \%)$ & - \\
& $286,3(14 \%)$ & $533,0(70 \%)$ & \\
& $288,0(5 \%)$ & $534,7(11 \%)$ & \\
& $284,8(80 \%)$ & - & \\
\hline
\end{tabular}

ligações químicas desde as formas de carbono oxidadas até átomos de carbono como produto final. O conteúdo em carbetos aumenta e, de acordo com os espectros Raman, são predominantemente de hibridização $\mathrm{sp}^{2}$.

Os espectros relacionados aos materiais tratados em 1000 e 2000 ${ }^{\circ} \mathrm{C}$ apresentam uma diminuição substancial na quantidade de heteroátomos. O nitrogênio já não é mais detectado acima de $1000{ }^{\circ} \mathrm{C}$, restando serem expulsos cerca de metade do oxigênio inicial (Figura 2). Os demais dados relacionados às ligações dos diversos elementos também se encontram na Tabela 1 e indicam uma diminuição na variedade de ligações químicas de todos os elementos, incluindo o carbono, ou seja, uma redução na variedade de grupos funcionais.

\section{Espectroscopia de espalhamento Raman}

A espectroscopia Raman é uma técnica útil para a caracterização da perfeição cristalina, porque o espalhamento Raman de cristais perfeitos é limitado às contribuições dos modos centrais da zona Raman-ativa. Em sistemas desordenados, o tamanho do cristalito torna-se muito menor que o comprimento de onda óptico, e a regra de seleção, que restringe o espalhamento Raman a modos essencialmente de centro de zona, é relaxada. ${ }^{20}$ Assim, para sistemas desordenados, as contribuições de outros fônons na primeira zona de Brillouin tornam-se possíveis, em particular nas frequiências onde há máximos na densidade de estados dos fônons e o acoplamento às radiações eletromagnéticas incidentes é significativo. Várias características nos espectros Raman de primeira e segunda ordem para carbono de caráter turbostrático podem, assim, ser usadas para caracterizar o carbono vítreo. Desta forma, uma correlação é obtida entre a temperatura de tratamento térmico e o grau de ordenamento estrutural que é alcançado no material. Para tanto, é importante definir o termo "turbostrático", sempre presente na análise de materiais carbonosos. Embora essa definição seja utilizada largamente na literatura, muitas vezes não é muito bem entendida ou explicada. Esse assunto é bastante complexo e, portanto, o próximo item mostra uma breve discussão dessa característica intrínseca dos materiais carbonosos, devido sua importância na discussão dos resultados. Uma discussão detalhada de espectroscopia de espalhamento Raman para materiais carbonosos pode ser obtida no recente trabalho de Gonçalves. ${ }^{21}$

Segundo Jenkins, ${ }^{22}$ pode-se definir o caráter turbostrático de um material carbonoso como o surgimento de superfícies não-planares ao longo das superfícies planares grafíticas. Entretanto, esse conceito é 
extremamente intuitivo e superficial para explicar todos os fenômenos que ocorrem nessa estrutura. Uma boa explicação de "turbostraticiade" pode ser iniciada a partir da estrutura da grafite pirolítico altamente orientado - Highly Oriented Pyrolitic Graphite (HOPG) - que é a mais próxima da grafite perfeita, ou seja, um empilhamento de lamelas planares, com átomos de carbono arranjados segundo estruturas grafênicas, cujas propriedades se repetem infinitamente. Cristalograficamente, a grafite monocristalina pertence ao grupo de simetria $\mathrm{D}_{4}{ }^{6 \mathrm{~h}}$, cuja célula unitária contém quatro átomos, dois de cada camada contribuinte, distanciados $0,142 \mathrm{~nm}$ nos planos e $0,3354 \mathrm{~nm}$ entre os planos, e os modos vibracionais são dos tipos $2 \mathrm{E}_{2 \mathrm{~g}}, 2 \mathrm{~B}_{2 \mathrm{~g}}, \mathrm{E}_{1 \mathrm{u}}$ e $\mathrm{A}_{2 \mathrm{u}}$. Costuma-se representar essa propriedade na Equaçã̃o $12^{23}$

$\Gamma=\mathrm{A}_{2 \mathrm{u}}+2 \mathrm{~B}_{2 \mathrm{~g}}+\mathrm{E}_{1 \mathrm{u}}+2 \mathrm{E}_{2 \mathrm{~g}}$

onde $\Gamma$ representa o vetor de onda de dispersão de fônons.

Somente dois modos $\mathrm{E}_{2 \mathrm{~g}}$ são ativos ao Raman e são identificados, um através da banda de Raman obtida a $1582 \mathrm{~cm}^{-1}$, e o outro através de espalhamento de nêutrons a baixa freqüência, em $47 \mathrm{~cm}^{-1}$. O HOPG exibe somente a banda $1582 \mathrm{~cm}^{-1}$ na região fundamental entre 1100 e $1700 \mathrm{~cm}^{-1}$, mas também mostra características de $2^{\mathrm{a}}$ ordem a 2400 e $3300 \mathrm{~cm}^{-1}$. A simetria dos modos $\mathrm{E}_{2 \mathrm{~g}}$ restringe-se ao movimento dos átomos no plano do carbono. Os dois diferentes modos $\mathrm{E}_{2 \mathrm{~g}}$ nesta análise tridimensional ocorrem porque os planos adjacentes podem vibrar em fase ou em fases opostas. ${ }^{24,25}$ Entretanto, o caso mais comum, para a estrutura dos carbonos de hibridização $\mathrm{sp}^{2}$, é o de anéis do tipo benzeno cujas propriedades se repetem dentro de dimensões bem definidas e limitadas. Costuma-se chamar esses casos de grafites microcristalinos, cujo caso mais extremo é o dos carbonos turbostráticos (que não se grafitizam, embora tendam a isso). A essas dimensões é dado o nome de tamanho de cristalito, $L_{a}$. Essas propriedades cristalográficas foram particularmente associadas aos dados de espectroscopia Raman, quando Tuinstra e Koenig ${ }^{24}$ encontraram uma importante relação entre o surgimento de variações na estrutura grafítica e a dimensão dos cristais. No espectro de primeira ordem, a mais importante variação induzida pela desordem é o aparecimento de um pico a $\sim 1360 \mathrm{~cm}^{-1}$, associado a uma larga densidade de estados de fônons.

A Figura 3 mostra um conjunto de espectros característicos de CVR-1000, CVR-1500 e CVR-2000 onde a evolução do comportamento das bandas Raman em função de HTT está bastante pronunciada. As bandas Raman-ativas do carbono vítreo reticulado

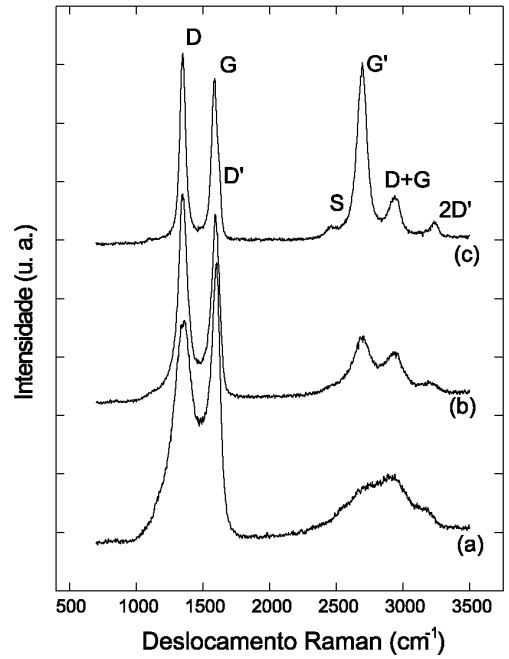

Figura 3. Espectros Raman de primeira e segunda ordem das amostras (a) CVR-1000, (b) CVR-1500 e (c) CVR-2000 estão indicadas no espectro (c) da Figura (CVR-2000), conforme a descrição: ${ }^{20,26} \mathrm{G}\left(1580 \mathrm{~cm}^{-1}\right)$ : está relacionado à extensão das estruturas que ocorrem de maneira repetitiva num mesmo cristal; D (1360 $\left.\mathrm{cm}^{-1}\right)$ : está relacionado à ruptura na repetibilidade das estruturas carbonosas envolvidas; D' $\left(1620 \mathrm{~cm}^{-1}\right)$ : contribuição associada aos diferentes tipos de defeitos estruturais; $\mathrm{S}\left(2450 \mathrm{~cm}^{-1}\right)$ : é uma banda de segunda ordem, descrita como um sobretom da banda em $~ 1220$ $\mathrm{cm}^{-1}$; G' $\left(2700 \mathrm{~cm}^{-1}\right)$ : banda de segunda ordem naturalmente presente em carbonos monocristalinos como o HOPG e considerada segunda ordem da banda D. Sua largura a meia altura esta associada ao nível de grafitização do material; $\mathrm{D}=\mathrm{D}+\mathrm{G}\left(2950 \mathrm{~cm}^{-1}\right)$ : modo de segunda ordem, ocasionado como combinação dos modos D e G; 2D' (3248 $\mathrm{cm}^{-1}$ ): modo de segunda ordem presente em carbono turbostrático com menor pronúncia do que a banda $\mathrm{D}$.

A banda D caracteriza uma forte desordem cristalina. Sua largura diminui à medida que aumenta HTT, sendo, portanto, mais estreita para CVR-2000 (Figura 3c). A largura da linha também indica a presença de outras influências sobre a desordem dentro das lamelas grafênicas, especialmente no que se refere à presença de impurezas, particularmente os heteroátomos. A partir dos espectros Raman é possível obter o comportamento das diferentes bandas com relação ao da banda $\mathrm{G}$, a qual é a única encontrada em HOPG para espectros de primeira ordem, e, portanto, considerada a referência do carbono mais organizado possível. Neste sentido, serão discutidas as intensidades relativas das bandas de primeira e segunda ordem do CVR, relativas à G, em função do HTT, como mostra a Figura 4. Adicionalmente, é também discutido o comportamento das respectivas larguras a meiaaltura dessas bandas.

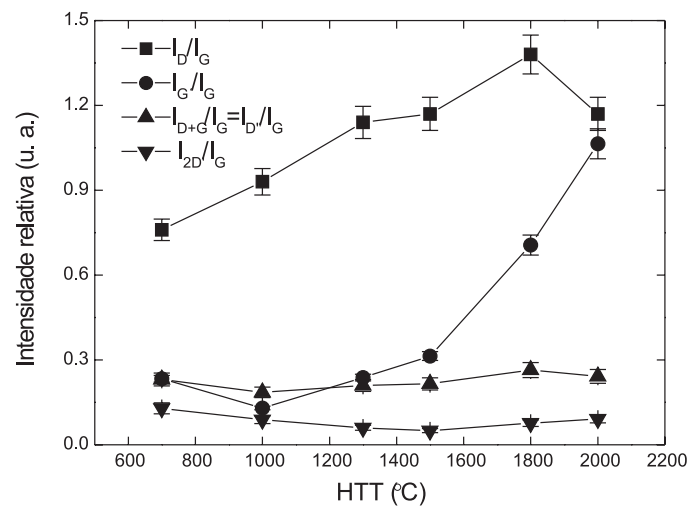

Figura 4. Comportamento das intensidades das diversas bandas Raman-ativas do CVR, com relação à intensidade da banda $G$, em função de HTT

A razão $I_{D} / I_{G}$ indica o quão importante é a contribuição direta dos defeitos na estrutura do material e está relacionada à formação da sua estrutura amorfa. Essa razão tem uma relação direta com $\mathrm{L}_{\mathrm{a}}$ (tamanho de cristalito) que indica o aumento da grafitização do CVR com aumento de HTT devido. Este comportamento não é objeto deste estudo e está descrito em detalhes em trabalho anterior. ${ }^{14} \mathrm{O}$ comportamento das bandas de segunda ordem associadas aos defeitos estruturais $\left(I_{\mathrm{D}+\mathrm{G}} / I_{\mathrm{G}}\right.$ e $\left.I_{2 \mathrm{D}} / / I_{\mathrm{G}}\right)$ é constante, mas com destaque no aspecto da curva que representa o comportamento de $I_{\mathrm{G}} / I_{\mathrm{G}}$. Entretanto, os valores decrescentes a largura a meia altura indicam que o material se torna monocristalino, como o HOPG. ${ }^{23}$ Esse dado pode ser importante para se avaliar o significado do decréscimo da razão $\left(I_{\mathrm{D}} / I_{\mathrm{G}}\right)$ após $1800^{\circ} \mathrm{C}$, relacionando-o à tendência de grafitização existente no decorrer do processo de tratamento térmico do material. No entanto, se considerarmos que o plano basal está mais curvo, com o aumento da temperatura, a energia elástica poderá ser liberada ocasionando a formação de planos prismáticos dando origem a defeitos pontuais, 
que ocasionam o decréscimo da razão $\left(I_{\mathrm{D}} / I_{\mathrm{G}}\right)$ após $1800^{\circ} \mathrm{C}$. No entanto, para constatar esse fato seria necessário assumir temperaturas mais elevadas no processo de tratamento térmico, ou seja, acima de $2000{ }^{\circ} \mathrm{C}$.

Uma outra variável relevante obtida a partir de espectroscopia Raman é a largura dos picos. Foi utilizada a definição de largura na meia-altura $(\omega)$ que é a medida da largura do pico correspondente à metade da intensidade do mesmo, a partir de sua deconvolução. A Figura 5 mostra as diferentes larguras em meia altura dos picos apresentados também em função de HTT, onde todas as bandas convergem para o comportamento de picos, pois sofrem significativo estreitamento. Entretanto, a banda 2D' se alarga em temperaturas mais baixas do intervalo. Primeiramente, é importante lembrar que a largura da banda está associada às razões pelas quais é formada. Uma banda mais estreita deve-se cada vez mais à melhor definição da banda e caracteriza o fato da contribuição que a origina estar cada vez mais pronunciada. Por exemplo, uma banda D muito intensa, mas muito estreita pode significar uma contribuição muito grande de defeitos, e estes, devidos especificamente à formação de um ou mais defeitos na estrutura evidenciando um modo de vibração especifico associado à presença do defeito. Por outro lado, uma banda mais larga pode indicar a soma de contribuições exógenas às características intrínsecas ao modo de vibração, como, por exemplo, a presença de heteroátomos.

Assim, ao final do processo de carbonização, todas as bandas indicam os efeitos obtidos a partir de sua descrição, e sua intensidade (especialmente a relativa à banda $\mathrm{G}$ ) indica sua específica contribuição. Entretanto, há um caso em que os defeitos podem ocorrer por tensionamento devido à presença de um átomo significativamente grande com relação ao carbono, como é o exemplo do nitrogênio e do oxigênio. A avaliação do comportamento de $\omega_{2 \mathrm{D}}$, fornece uma importante informação do que acontece na faixa crítica de temperatura
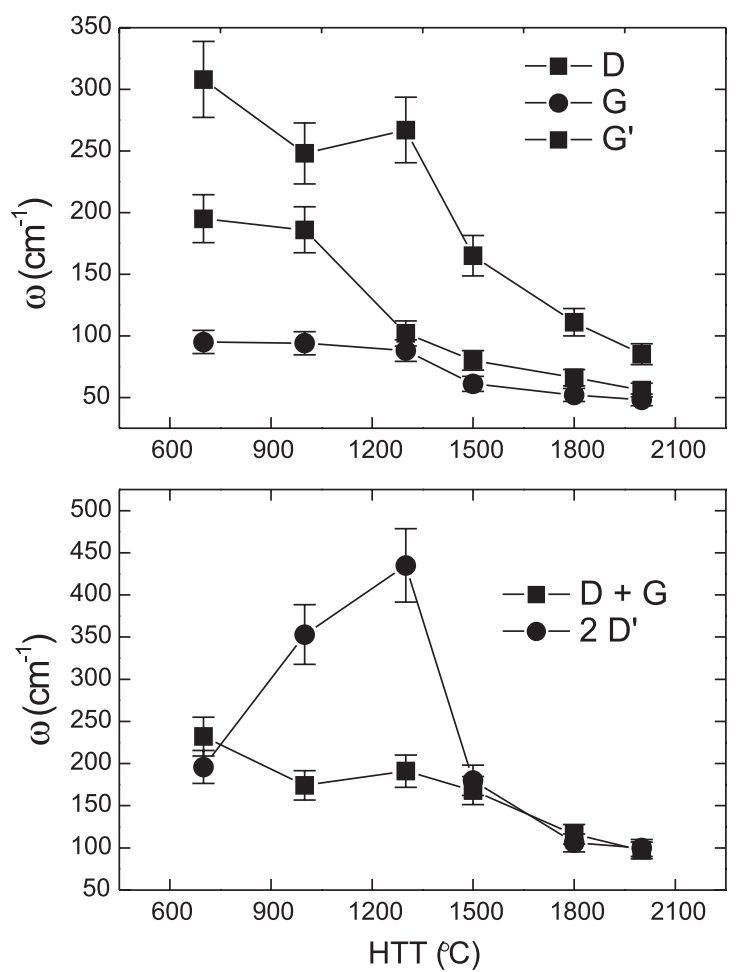

Figura 5. Comportamento da largura na meia-altura das diversas bandas do CVR em função de HTT. Os gráficos foram divididos em 2 conjuntos para facilitar a visualização de $1300{ }^{\circ} \mathrm{C}$, onde os últimos resquícios de heteroátomos são expelidos do material. Observe-se que $\omega_{2 \mathrm{D}}$, cresce significativamente em HTT vizinhas a $1300^{\circ} \mathrm{C}$. Esse mesmo efeito dificulta a grafitização observada em $\mathrm{G}$ e em G': a partir dessa temperatura, seus valores de $\omega$ caem de maneira intensa. Destaca-se, pois, o fato de que o estreitamento das bandas está intimamente relacionado à expulsão de heteroátomos. Justamente pelo fato de o precursor do carbono vítreo reticulado ser composto de polímeros nitro- e oxigenados, todas as bandas se iniciam muito largas, estreitando-se à medida que o material é carbonizado, em temperaturas superiores a $1300^{\circ} \mathrm{C}$. Esta temperatura se torna um limite, pois, para se definir a presença total de carbono e para se associar os fenômenos observados em espectroscopia Raman intrinsicamente ao material carbonoso.

\section{Voltametria cíclica}

Uma série de experimentos foi conduzida para estudar o comportamento de eletrodos de CVR em par redox ferri/ferrocianeto. $\mathrm{O}$ principal objetivo foi verificar a coerência da resposta dos eletrodos usando alguns critérios de quasi reversibilidade para as reações redox usuais neste eletrólito. ${ }^{25}$ A correlação entre os resultados das análises morfológica e estrutural previamente discutida e os dados voltamétricos dão consistência a este estudo. A resposta eletroquímica também permitiu avaliar a área de superfície eletroquímica específica (Surface Electrochemical Specific Area - SESA) dos eletrodos de CVR. Os resultados mostraram uma forte dependência entre a HTT e o comportamento da reversibilidade, como esperado. Este método representa uma técnica adicional para o controle de qualidade do eletrodo de CVR, onde a seguinte notação foi usada: $S_{s}$ para a área de superfície específica $\left(\mathrm{cm}^{2} \mathrm{~cm}^{-3}\right) ; I_{p}$ para a intensidade de corrente do pico anódico (A); $C$ para a concentração do oxidante (idealizada como sendo igual à do redutor); $V_{e l}$ para o volume de eletrodo imerso na solução $\left(\mathrm{cm}^{3}\right) ; D_{o}$ para a difusividade do oxidante na solução $\left(\mathrm{cm}^{2} \mathrm{~s}^{-1}\right.$, também considerada igual à do redutor), e $v$ para a taxa de varredura de tensão em experimentos voltamétricos $\left(\mathrm{V} \mathrm{s}^{-1}\right)$.

Os estudos voltamétricos mostraram que os eletrodos de CVR podem ser analisados pelos critérios de quasi-reversibilidade. Esta análise foi associada com os parâmetros cinéticos $\Delta E_{\mathrm{p}}$ e $I_{\mathrm{Pc}_{\mathrm{c}}}$ como função de HTT e velocidade de varredura. ${ }^{8}$ Para todos os eletrodos, foi observado que, em geral, o aumento da taxa de varredura promove um aumento na intensidade da corrente de pico nas reações catódicas e anódicas (Figura 6a). O comportamento tende à irreversibilidade quando a temperatura de tratamento térmico aumenta. De modo particular, os eletrodos CVR 1300 e CVR 1500 apresentam valores maiores de $\Delta E_{\mathrm{p}}$. Este fato é compreensível se devidamente observado o fato da singularidade da temperatura de $1300{ }^{\circ} \mathrm{C}$ no que se refere ao seu comportamento estrutural, bem como o intervalo subseqüente de HTT. Trata-se do fim do regime de influência dos heteroátomos e grupos funcionais e do início do regime puramente associado ao ordenamento da estrutura carbonosa. Dentre o intervalo do carbono puro, o CVR 1300 é o que apresenta maior índice de defeitos, além de uma banda 2D' intensa, ainda fortemente associada às impurezas anteriormente presentes no material. Especificamente, entre 1300 e $1500{ }^{\circ} \mathrm{C}$ inicia-se o reordenamento da estrutura, livre de grupos funcionais.

Outro aspecto muito importante a ser destacado é o comportamento da área de superfície eletroquímica específica (SESA), que é determinada pela Equação de Randles-Sevčik: ${ }^{8}$

$\mathrm{SESA}=\frac{I_{p}}{2,69 \times 10^{5} C_{o} V_{e l} \sqrt{D_{o} v}}$

Os valores de SESA são mostrados na Figura 6b. Estes valores 

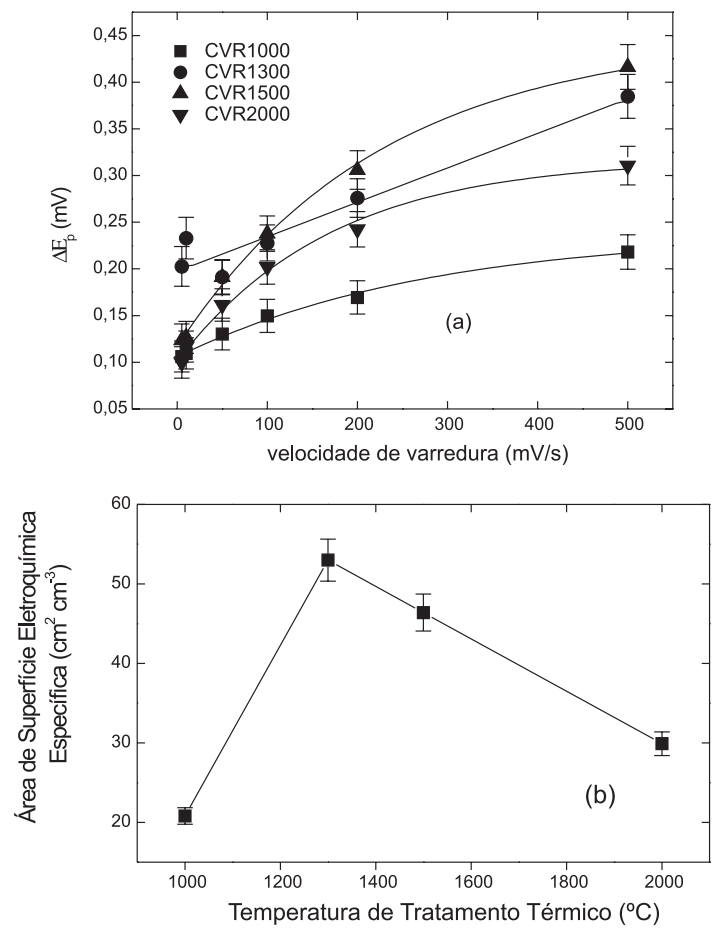

Figura 6. (a) Comportamento de $\Delta E_{p}$ em função da velocidade de varredura de voltametria em par redox ferri-ferrocianeto para eletrodos CVR obtidos em diferentes HTT; (b) área de superfície eletroquímica específica (SESA) do CVR em função de HTT

estão em excelente acordo com as singularidades já encontradas para o material no intervalo de temperatura de tratamento térmico observado. As HTT mais baixas proporcionam maior efeito resistivo, o que é determinante para a resposta eletroquímica do eletrodo. Além disso, a influência da corrente medida pode também conter uma contribuição desconhecida proveniente de modificações da superfície do eletrodo. ${ }^{27} \mathrm{O}$ máximo encontrado para SESA do CVR 1300 está em forte correlação com o comportamento dos índices Raman $I_{\mathrm{D}} / I_{\mathrm{G}}$, $I_{\mathrm{D}+\mathrm{G}} / I_{\mathrm{G}}$ e $I_{2 \mathrm{D}}, / I_{\mathrm{G}}$, uma vez que a largura e a intensidade dessas bandas encontram mudança de comportamento nessa temperatura. Além disso, é justamente nesta temperatura que o material apresenta um novo aumento da distância interlamelar, revelando falta de simetria grafítica e intenso caráter turbostrático. ${ }^{12}$ Dessa forma, esse comportamento reflete as propriedades já estudadas ${ }^{8}$ de $\mathrm{HOPG}$ oxidado em carbonos de borda, que, com o surgimento de defeitos, proporcionados por esse tratamento químico, aumentam a velocidade transferência de elétrons e o número de sítios associados a esses processos.

\section{CONCLUSÕES}

O acompanhamento de importantes propriedades físico-químicas do carbono vítreo reticulado pôde ser realizado pelas técnicas aqui propostas, além de relacioná-las com algumas propriedades eletroquímicas. A técnica Raman mostrou-se de central relevância nas análises que dizem respeito à evolução da estrutura do CVR com o HTT, pois as análises superficiais e eletroquímicas foram relacionadas aos modos de vibração de primeira e segunda ordem do material obtido nas diversas temperaturas de tratamento térmico do intervalo estudado. Foi possível, inclusive, estimar um valor limite de temperatura, em $1300^{\circ} \mathrm{C}$, para as mudanças que influenciam nas diversas propriedades, ou seja, a dependência entre a presença de heteroátomos e os modos de vibração do material. A contribuição deste trabalho foi mostrar como os mecanismos da formação estrutural do material estão rela- cionados neste intervalo de HTT. Embora o material já apresente uma característica tipicamente condutora a partir de $1000^{\circ} \mathrm{C}$, somente em $1300{ }^{\circ} \mathrm{C}$ é possível definir um material carbonoso propriamente dito. Essa informação é de valiosa importância nas propriedades anódica e catódica do mesmo, regulada pela acidez ou basicidade dos grupos funcionais presentes, bem como da molhabilidade ou da adesão de filmes nos diferentes processos onde o CVR pode ser utilizado como substrato. Assim, os grupos funcionais de superfície, especialmente os oxigenados, regulam as propriedades desse material até a temperatura de $1300{ }^{\circ} \mathrm{C}$, sendo que nitrogênio apresenta importância abaixo de $1000^{\circ} \mathrm{C}$, enquanto acima disso, a empilhamento das lamelas grafênicas e os índices de defeitos e ainda a turbostraticidade do carbono determinam os modos de vibração observados nos espectros Raman de primeira e segunda ordem. Também, nas análises de VC, foi observada uma forte relação entre os índices de caráter turbostrático e defeitos, analisados por Raman, o caráter funcional, analisados por XPS e, ainda, a resposta eletroquímica do CVR.

\section{AGRADECIMENTOS}

Ao CNPq (Processos 301583/06-3 e 305009/2006-0) e Fapesp (Processo 05/50718-9) pelo apoio financeiro. Agradecimentos especiais ao Prof. Dr. P. A. P. Nascente da UFSCar pelas análises de XPS.

\section{REFERÊNCIAS}

1. Rezende, M. C.; Tese de Doutorado, Universidade de São Paulo, Brasil, 1991.

2. Kaplan, R. B.; US pat. 5,282,861 1994.

3. Wutor, V. C.; Togo, C. A.; Limson, J. L.; Pletschke, B. I.; Enz. Microb. Technol. 2007, 40, 1512.

4. Goldblum, D. K.; Holodnick, S. E.; Mancy, K. H.; Briggs, D. E.; Environ. Prog. 2006, 10, 24.

5. Tentorino, A.; Casolo-Ginelle, U.; J. Appl. Electrochem. 1978, 8, 195.

6. Bowling, R. J.; Packard, R. T.; McCreery, R. L.; J. Am. Chem. Soc. 1989, 111,1217

7. Wang, Y.; Alsmeyer, D. C.; McCreery, R. L.; Chem. Mater. 1990, 2, 557.

8. McCreery, R. L. Em Electroanalytical Chemistry; Bard, A. J., ed.; Dekker: New York, 1991.

9. Gerischer, H.; Heller, A.; J. Phys. Chem. 1991, 95, 5261.

10. McDermott, M. T.; Kneten, K.; McCreery, R. L.; J. Phys. Chem. 1992, 96, 3124.

11. Gonçalves, E. S.; Rezende, M. C.; Takahashi, M. F. K.; Ferreira, N. G.; Mater. Res. 2006, 9, 147.

12. Gonçalves, E. S.; Rezende, M. C.; Ferreira, N. G.; Braz. J. Phys. 2006, $36,264$.

13. Gonçalves, E. S.; Dalmolin, C.; Biaggio, S. R.; Nascente, P. A. P.; Rezende, M. C.; Ferreira, N. G.; Appl. Surf. Sci. 2007, 253, 8344.

14. Baldan, M. R.; Almeida, E. C.; Azevedo, A. F.; Gonçalves, E. S.; Rezende, M. C.; Ferreira, N. G.; Appl. Surf. Sci. 2007, 254, 600.

15. Baldan, M. R.; Ramos, S. C.; Almeida, E. C.; Azevedo, A. F.; Ferreira, N. G.; Diamond Rel. Mat. 2008, 17, 1110.

16. Moulder, J. F.; Stickle, W. F.; Sobol, P. E.; Bomben, K. D. Em Handbook of X-ray Photoelestron Spectroscopy; Praire, E., ed.; Pelkin-Elmer Corporation Physical Electronics Division: Minessota, 1992.

17. Jiang, Y. B.; Zhang, H. X.; Cheng, D. J.; Yang, S. Z.; Thin Solid.Films 2000, 360, 52

18. Quirós, C.; Núñez, R.; Prieto, P.; Vergara, I.; Cáceres, D.; Soriano, L.; Fuentes, G. G.; Elizalde, E. J.; Sanz, M.; Surf. Coat. Technol. 2000, 125, 284.

19. Gammon, W. J.; Kraft, O.; Reilly, A. C.; Holloway, B. C.; Carbon 2003, 
41, 1917.

20. Chieu, T. ; Dresselhaus, M. S.; Endo, M.; Phys. Rev. B. 1982, 26, 5867.

21. Gonçalves, E. S.; Tese de Doutorado, Instituto Tecnológico da Aeronáutica, Brasil, 2007.

22. Jenkins, G. M.; Kawamura, K.; Polymeric Carbons - Carbon Fibre, Glass and Char, Cambridge University Press: Cambridge, 1976.

23. Mani, K. K.; Ramani, R.; Phys. Solid State. 1974, 61, 659.

24. Tuinstra, F.; Koenig, J. L.; J. Chem. Phys. 1970, 53, 1126.
25. Greef, R.; Peat, R.; Peter, L. M.; Pletcher, D.; Robinson, J.; Instrumental Methods in Electrochemistry, Ellis Horwood Limited/John Wiley and Sons: New York, 1985.

26. Shimada, T.; Sugai, T.; Fantini, C.; Souza, M.; Cançado, L. G.; Jorio, A.; Pimenta, M. A.; Saito, R.; Grüneis, A.; Dresselhaus, G.; Dresselhaus, M. S.; Ohno, Y.; Mizutanih T.; Shinohara, H.; Carbon 2005, 43, 1049.

27. Leon y Leon, C. A.; Radovic, L. R. Em Interfacial Chemistry and Electrochemistry of Carbon Surfaces; Thrower, P. A., ed.; Dekker: New York, 1994. 